\title{
Dynamics of connective-tissue localization during chronic Borrelia burgdorferi infection
}

\author{
Denise M Imai, Sunlian Feng, Emir Hodzic and Stephen W Barthold
}

The etiologic agent of Lyme disease, Borrelia burgdorferi, localizes preferentially in the extracellular matrix during persistence. In chronically infected laboratory mice, there is a direct association between $B$. burgdorferi and the proteoglycan decorin, which suggests that decorin has a role in defining protective niches for persistent spirochetes. In this study, the tissue colocalization of B. burgdorferi with decorin and the dynamics of borrelial decorin tropism were evaluated during chronic infection. Spirochetes were found to colocalize absolutely with decorin, but not collagen I in chronically infected immunocompetent $\mathrm{C} 3 \mathrm{H}$ mice. Passive immunization of infected $\mathrm{C} 3 \mathrm{H}$-scid mice with $\mathrm{B}$. burgdorferi-specific immune serum resulted in the localization of spirochetes in decorin-rich microenvironments, with clearance of spirochetes from decorin-poor microenvironments. In passively immunized $\mathrm{C} 3 \mathrm{H}$-scid mice, tissue spirochete burdens were initially reduced, but increased over time as the B. burgdorferi-specific antibody levels waned. Concurrent repopulation of the previously cleared decorin-poor microenvironments was observed with the rising tissue spirochete burden and declining antibody titer. These findings indicate that the specificity of $B$. burgdorferi tissue localization during chronic infection is determined by decorin, driven by the borrelia-specific antibody response, and fluctuates with the antibody response.

Laboratory Investigation (2013) 93, 900-910; doi:10.1038/labinvest.2013.81; published online 24 June 2013

KEYWORDS: Borrelia burgdorferi; decorin; decorin-binding proteins; Lyme disease; mouse model

Borrelia burgdorferi, the causative agent of Lyme borreliosis, is a tick-transmitted spirochete that establishes chronic extracellular infection in mammalian hosts. ${ }^{1,2}$ Lyme borreliosis progresses through multiple loosely defined stages, including local infection, dissemination, and persistence. ${ }^{1}$ During initial tick-borne infection, spirochetes are deposited within the dermis, locally proliferate, and then disseminate to distant tissues. ${ }^{3-5}$ Distant dissemination is followed by or occurs simultaneously with the onset of acquired immunity, which resolves disease (ie, arthritis and carditis) and reduces the number of spirochetes in tissue. ${ }^{6}$ Remaining spirochetes that have evaded the acquired immune response persist for months to years. ${ }^{7-10}$ At each of these stages, morphologically intact spirochetes are identifiable in the extracellular matrix (ECM). During early infection, disseminating spirochetes colonize a broad range of tissues. ${ }^{1,3,6}$ During chronic infection, persisting spirochetes are restricted to the ECM of the dermis, periarticular connective tissue, tendons, ligaments, perineurium, periaortic connective tissue at the base of the heart, and other collagen-rich sites. $3,4,6,7,11-13$
Since the initial disease description, spirochetes have been described as aligning with or intercalated between collagen fibers in the ECM of connective tissue. ${ }^{3,6,7,14}$ Collagen is but one component of the molecularly diverse ECM. The ECM is composed of a network of fibrillar proteins embedded within a hydrated ground substance composed of proteoglycans and glycosaminoglycans. ${ }^{15}$ In order to colonize these connective tissue-rich predilection sites, any bacterium, including a spirochete, must interact with these various ECM components through bacterial cell surface adhesins. ${ }^{16}$ B. burgdorferi encodes a surprisingly large number of ECM adhesins for its relatively small genome (reviewed by Antonara et $a l^{17}$ ). The known borrelial ECM adhesins include decorin-binding protein (Dbp)A and B, Borrelia glycosaminoglycan-binding protein (Bgp), fibronectinbinding protein (BBK32), RevA, RevB, members of the Borrelia membrane protein family (BmpA-D), OspEF-related proteins (Erps), P66, and a yet to be identified adhesin that binds directly to type I collagen. These adhesins bind respectively to decorin, various glycosaminoglycans, 
fibronectin, laminin, and $\alpha_{\mathrm{IIb}} \beta_{3}$ and $\alpha_{\mathrm{v}} \beta_{3}$ integrins. ${ }^{17-25}$ Based on the number of putative borrelial adhesins, many more borrelial adhesin-ECM ligand interactions are likely to be revealed in future. ${ }^{17,26}$

Given the wealth of adhesins elaborated by $B$. burgdorferi, it is not surprising that no single adhesin is absolutely essential to infection and persistence. ${ }^{2,17}$ Lack of a single adhesin can alter pathogenicity by influencing specific stages of disease. For example, DbpA/B and decorin is one adhesinligand interaction that has been scrutinized in relation to chronic infection. Liang et $a^{27}$ observed a direct relationship between the numbers of persisting spirochetes and decorin expression level in collagen-rich tissues (eg, skin, joint, and heart). The observed protective capacity of these tissues was specific to decorin, evident only in the face of an acquired immune response, and associated with an increased expression of $d b p A .^{27}$ In chronic (post-disease resolution) infection, Barthold et $a l^{6}$ demonstrated that the antibodymediated immune response resulted in a change in the microenvironmental localization of persisting spirochetes into collagen-rich connective tissues. Together, these observations support the existence of preferential connectivetissue localization during chronic Lyme borreliosis. They further suggest that preferential connective-tissue localization may be driven by the humoral immune response and that decorin may be a major determining ECM component.

The purpose of this study was to evaluate the microenvironmental dynamics of connective-tissue localization in chronic stages of Lyme borreliosis in the mouse model, evaluate the influence of the B. burgdorferi-specific antibody response on the specificity of tissue localization, and evaluate the extent to which decorin determines connective-tissue localization. We focused on the heart and, in particular, the connective tissue of the heart base, as a model tissue for study, as it is a consistent site into which disseminating spirochetes colonize and a site in which inflammation (carditis) develops during the early phase of infection., ${ }^{3,4,11}$ After induction of the immune response, the heart base is also a site in which disease resolves, but spirochetes persist within connective tissue. ${ }^{6,7}$ Additionally, the heart base contains closely apposed decorin-rich (vascular adventitia) and decorin-poor (vascular media) microenvironments that other tissues, such as joints, lack. An added advantage of focusing on the heart base is that decalcification, which can result in inadequate antigen preservation, is not necessary. Using the heart in a well-characterized $\mathrm{C} \mathrm{H}^{7}$ and passively immunized C3H-scid ${ }^{6,27-29}$ mouse model of chronic Lyme borreliosis, the present study confirmed the preferential connective-tissue localization of $B$. burgdorferi and demonstrated that preferential connective-tissue localization is specific to decorin-rich microenvironments, is driven by a $B$. burgdorferi-specific antibody response, and that recrudescence results in loss of decorin-specific connectivetissue localization.

\section{MATERIALS AND METHODS \\ Borrelial Strains}

The low passage B. burgdorferi 'sensu stricto' strains cN40 and B31were utilized to establish infections. ${ }^{7,30,31}$ B. burgdorferi cultures were grown in liquid modified Barbour-StoennerKelly (BSKII) medium supplemented with 6\% normal rabbit serum. $^{32}$

\section{Mice}

Specific-pathogen-free, 3-5-week-old, female $\mathrm{C} 3 \mathrm{H} / \mathrm{HeN}$ $(\mathrm{C} 3 \mathrm{H})$ mice were acquired from Frederick Cancer Research Center (Frederick, MD, USA) and severe combined immunodeficient C3H/Smn.CIcrHsd-Prkdc scid (C3H-scid) mice were obtained from Harlan Sprague Dawley (Indianapolis, IN, USA). Mice were killed by carbon dioxide narcosis and cardiac exsanguination.

\section{Experimental Infections}

Mice were infected by subdermal inoculation of $10^{4}-10^{5}$ mid-log phase B. burgdorferi in $0.1 \mathrm{ml}$ BSKII medium on the dorsal thoracic midline. To represent naive mice, uninfected mice were necropsied without borrelial inoculation. To represent chronic infection, $\mathrm{C} 3 \mathrm{H}$ mice were necropsied at day 60 post inoculation. For passive immunization studies to manipulate humoral immunity, immune serum from $\mathrm{C} 3 \mathrm{H}$ mice infected with either B. burgdorferi $\mathrm{cN} 40$ or B31 was collected at 60 days of infection, as previously described. ${ }^{29}$ Infected $\mathrm{C} 3 \mathrm{H}$-scid mice were passively immunized with $0.3 \mathrm{ml}$ immune serum subcutaneously on days 12,18 and 24 post inoculation. Immune serum administered was homologous to the specific B. burgdorferi strain ( $\mathrm{cN} 40$ or B31) used to establish infection. Negative controls were administered normal mouse serum (NMS) from uninfected naive mice. Subsets of passively immunized $\mathrm{C} 3 \mathrm{H}$-scid mice were necropsied on days 28,56 and 72 post inoculation. Sub-inoculation site and urinary bladder tissues were aseptically collected for culture to confirm infection, as previously described. ${ }^{7}$ Tissues collected for DNA extraction included skin, heart base, ventricular muscle, quadriceps muscle, and left tibiotarsus. Tissues collected for immunohistochemistry (IHC) and immunofluorescence (IF) included heart base and myocardium. Hearts were bisected along the longitudinal axis to provide samples for both DNA extraction, IHC and IF. Mice used in infectivity experiments that were neither culture- nor quantitative PCR (qPCR)-positive were discarded from the data set.

\section{Quantitative PCR}

DNA was extracted from tissues using DNeasy tissue kits, according to the manufacturer's instructions (QIAGEN, Valencia, CA, USA). Samples were analyzed by qPCR for B. burgdorferi flagellin (flaB) DNA, using a previously optimized primer set and internal hydrolysis probe assay ${ }^{33}$ to confirm infection and quantify tissue spirochete burdens. Quantification of gene copies was based on absolute standard 
curves prepared using plasmid standards. ${ }^{33}$ Target-gene copy numbers were expressed as copy number of flaB per mg of tissue weight.

\section{Enzyme-Linked Immunosorbent Assay}

Ninety-six-well plates were coated with $1 \mu \mathrm{g} / \mathrm{ml} \mathrm{B}$. burgdorferi cN40 whole-cell lysate or $0.5 \mu \mathrm{g} / \mathrm{ml}$ rabbit anti-mouse IgG antibody (Southern Biotech, Birmingham, AL, USA) in carbonate coating buffer ( $\mathrm{pH}$ 9.6), as described previously. ${ }^{34}$ The secondary antibody used was alkaline phosphataseconjugated goat anti-mouse IgG, diluted at 1:5000 (Jackson ImmunoResearch Laboratories Inc., West Grove, PA, USA). Antibody-binding was revealed using $1 \mathrm{mg} / \mathrm{ml}$ phosphate substrate (Sigma-Aldrich, St Louis, MO, USA) in diethanolamine buffer. Optical density values $(405 \mathrm{~nm})$ were measured on a kinetic microplate reader (Molecular devices, Sunnyvale, CA, USA), as described previously. ${ }^{6}$ Immune sera and individual mouse serum samples from passively immunized $\mathrm{C} 3 \mathrm{H}$-scid mice were titrated in threefold dilutions (starting at 1:300) for immunoreactivity to either B. burgdorferi $\mathrm{cN} 40$ or for total serum IgG levels. All serum samples were tested in duplicate, and each assay included uninfected mouse serum (NMS from $\mathrm{C} 3 \mathrm{H}$ and/or $\mathrm{C} 3 \mathrm{H}$-scid mice, as needed) as a negative control and day 60 -post inoculation $B$. burgdorferi cN40-infected mouse immune serum as a positive control.

\section{Immunofluorescence}

Tissues were embedded in Tissue-Tek OCT compound (Sakura, Torrance, CA, USA), flash-frozen in liquid nitrogen and stored at $-80^{\circ} \mathrm{C}$. Tissues were sectioned $5-\mu \mathrm{m}$ thick on Superfrost/Plus slides (Thermo Fisher Scientific, Waltham, MA, USA), fixed for $5 \mathrm{~min}$ in cold acetone, air-dried for $30 \mathrm{~min}$ and rehydrated in PBS for $10 \mathrm{~min}$. Non-specific reactivity was blocked by incubation with $10 \%$ horse serum in PBS for $30 \mathrm{~min}$. Tissue sections were incubated with primary goat anti-decorin antibody (R\&D systems, Minneapolis, MN, USA) for $12 \mathrm{~h}$ at $4{ }^{\circ} \mathrm{C}$. Primary antibody reactivity was assessed by incubation with the appropriate NorthernLights 493 fluorochrome-labeled secondary donkey anti-goat antibody (R\&D systems) for $1 \mathrm{~h}$ at room temperature. Rinses were performed in PBS with $0.05 \%$ Tween 20 (SigmaAldrich) to reduce surface tension. Tissues were counterstained with DAPI for 10 min, mounted in Fluoromount-G (Southern Biotech, Birmingham, AL, USA) and cover-slipped.

\section{Histochemistry and IHC}

Tissues were immersion-fixed in 10\% neutral-buffered formalin, processed, paraffin-embedded, and sectioned $5-\mu \mathrm{m}$ thick onto Superfrost/Plus slides. Prior to processing, the heart base aorta was appropriately oriented in HistoGel (Thermo Scientific Richard-Allan Scientific, Kalamazoo, MO, USA). Sections of heart base (including aorta) were stained for connective tissue with Masson's trichrome. Sections of heart base (including aorta) were evaluated for the presence of B. burgdorferi, as described previously ${ }^{29}$ with the following modifications. Sections were incubated with the following primary antibodies, polyclonal immune serum from B. burgdorferi-infected rabbits or polyclonal rabbit anti-collagen I antibody (Abcam, Cambridge, MA, USA) or goat anti-decorin antibody (R\&D systems), for $12 \mathrm{~h}$ at $4{ }^{\circ} \mathrm{C}$. The secondary goat anti-rabbit EnVision + system-HRP (DAKO, Carpinteria, CA, USA) antibody was pre-conjugated to a non-avidin/biotin horseradish peroxidase-labeled polymer. A secondary anti-goat streptavidin-biotin immunoenzymatic detection system (Goat Cell and Tissue Staining Kit, R\&D systems) was utilized, per manufacturer's instructions. Rinses were performed in PBS with $0.05 \%$ Tween 20 to reduce surface tension. Each immunohistochemical run was performed with the inclusion of a negative (by omission of the primary antibody) and positive (tissues from infected $\mathrm{C} 3 \mathrm{H}-$ scid mice in which spirochetes were previously identified by IHC) control.

Sagittal sections through the heart, including the aorta at the heart base, were examined for the presence of spirochetes in the heart base and ventricular myocardium in the following microenvironments: tunica adventitia (connective tissue surrounding arteries), tunica media (the smooth muscle wall of arteries), and myocardial connective tissue. The myocardial connective tissue microenvironment included the myocardial interstitium, epicardium and connective tissue at the heart base (not associated with the great vessels). The anatomic sites and microenvironments were defined based on previous observations of borrelial distribution in the cardiovascular system. ${ }^{4,11,29}$ Numbers of spirochetes within anatomic locales were graded on a scale of 0 (no spirochetes), 0.5 (rare spirochetes), 1 (small numbers), 2 (moderate numbers), and 3 (large numbers). The mean score \pm standard error (s.e.m.) was calculated for each treatment group. Tissue sections were blindly examined, without knowledge of treatment group, by a single pathologist.

\section{Image Acquisition}

Epifluorescent and brightfield images were acquired on a Zeiss Axioskop microscope with a Zeiss AttoArc HBO $100 \mathrm{~W}$ mercury lamp, Zeiss filter sets, Zeiss Axiocam camera and Zeiss Axiovision software. All image manipulations, including resizing, white-balance adjustment, brightness adjustment (equivalent across epifluorescent images), arrowheads and added text were performed in Adobe Photoshop CS4.

\section{Statistics}

Analyses were performed using independent samples $t$-test or one-way analysis of variance, followed by post hoc pair-wise comparisons (Tukey's HSD test) (Prism v. 5, GraphPad software). Calculated $P$-values $\leq 0.05$ were considered significant. 


\section{RESULTS}

Localization of B. burgdorferi in the Tunica Adventitia and Myocardial Connective Tissue During Chronic Infection

To begin evaluation of spirochetal connective-tissue localization during chronic infection, the specific cardiovascular microenvironments in which $B$. burgdorferi persist were investigated. Borrelial infection was maintained in 10 immunocompetent $\mathrm{C} 3 \mathrm{H}$ mice inoculated with $B$. burgdorferi strain $\mathrm{cN} 40$ for 60 days, a time point at which immunemediated disease resolution and reduction of spirochete burdens in tissues has occurred. ${ }^{33}$ Distribution of spirochetes within (i) anatomic sites (heart base versus ventricular myocardium) and (ii) microenvironments (tunica adventitia, tunica media, and myocardial connective tissue) was evaluated by IHC. A very small number of spirochetes were found (Figure 1) randomly distributed throughout the heart. Though no anatomic site specificity (heart base versus myocardium) was observed, microenvironmental specificity was apparent. The only microenvironments in which spirochetes were identified were in the tunica adventitia (Figure 1a) and myocardial connective tissue (Figure 1b). No spirochetes were identified within the tunica media.

\section{Spirochete Localization in the Tunica Adventitia and Myocardial Connective Tissue is Induced by the B. Burgdorferi-Specific Antibody Response}

The original descriptions of spirochete distribution after antibody-mediated disease resolution ${ }^{6,27,29,35}$ were made using strain N40 or B31. However, the microscopic localization of morphologically intact spirochetes has only been reported with B. burgdorferi N40. DbpA-N40 and DbpA-B31 have only an $81 \%$ amino-acid sequence similarity, ${ }^{36}$ with variable in vitro decorin-binding abilities. ${ }^{37,38}$ Thus, the influence of the DbpA/B-decorin interaction and connective-tissue localization could potentially be strain-specific. Therefore, evaluation of spirochete localization post disease resolution was performed using strain B31, to compare with the N40 archetype in order to account for possible strain-specific variation in microenvironmental localization. ${ }^{6}$

To evaluate the contribution of humoral immunity to the microenvironmental specificity (and potential preferential connective-tissue localization) of borrelial persistence, 10 immunodeficient $\mathrm{C} 3 \mathrm{H}$-scid mice were inoculated with B. burgdorferi strain B31. A subset of five mice was passively immunized with B31-specific immune serum and a subset of five mice was administered serum from uninfected mice (NMS). At necropsy on day 28 post inoculation, a very small number of spirochetes were identified within the tunica adventitia and myocardial connective tissue (Table 1) in the mice treated with B31-specific immune serum, in contrast to mice treated with NMS, in which spirochetes could be identified in all microenvironments. Relative spirochete burden scores in these microenvironments were statistically significantly lower in mice treated with immune serum (all $P$-values $\leq 0.05)$ than in the heavily colonized C3H-scid mice administered NMS (Table 1). The tunica media of $\mathrm{C} 3 \mathrm{H}$-scid mice treated with immune serum was cleared of spirochetes, while the tunica media of mice administered NMS was not. Thus, in passively immunized $\mathrm{C} 3 \mathrm{H}$-scid mice, the distribution of spirochetes on day 28 recapitulated the distribution of persistent spirochetes in immunocompetent mice, the distribution of persistent N40 spirochetes, and reaffirmed the accuracy with which passive immunization reproducibly induces antibody-mediated tissue spirochete reduction and microenvironmental location for persistence. ${ }^{27-29,35}$ The data supported the hypothesis that microenvironment-specific localization of persistent spirochetes is associated with a B. burgdorferi-specific antibody response.
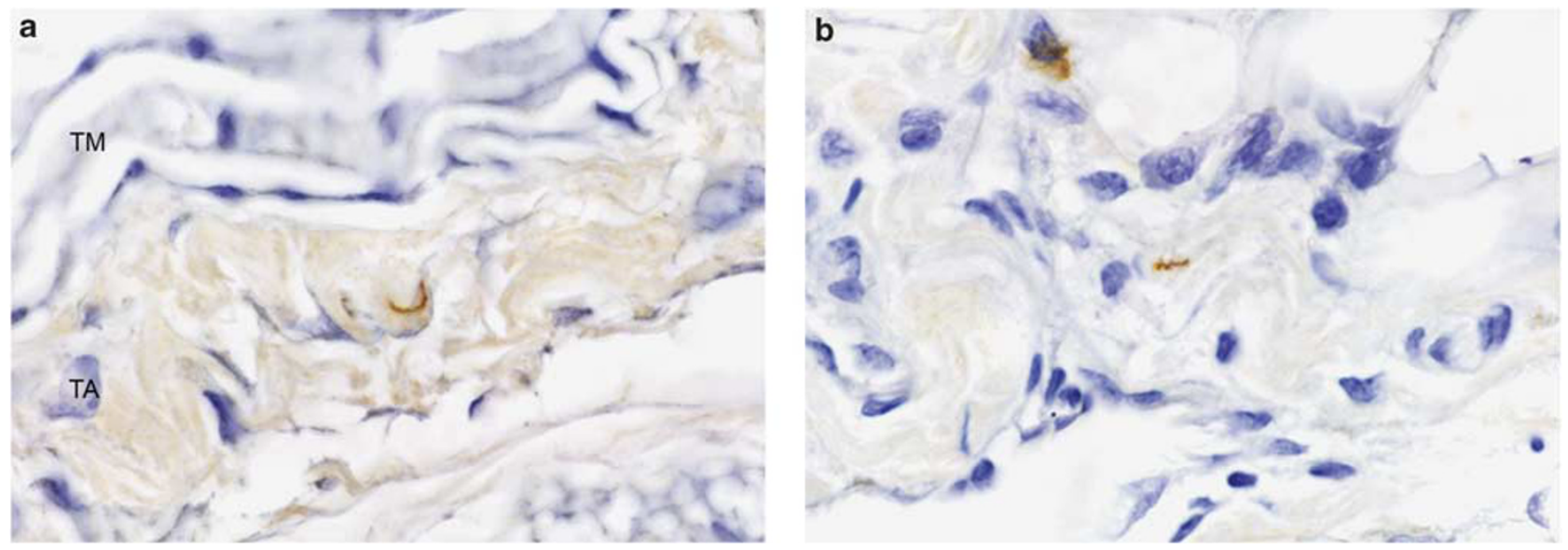

Figure 1 In chronic infection, spirochetes localized to the tunica adventitia (TA) (a) and myocardial connective tissues (b) in the cardiovascular tissue of a $\mathrm{C} 3 \mathrm{H}$ mouse, 60 days post inoculation with B. burgdorferi. The tunica media (TM) was devoid of spirochetes. Indirect IHC with rabbit anti-B. burgdorferi immune serum and immunoperoxidase reaction with 3, 3'-diaminiobenzidine (DAB) substrate; hematoxylin counterstain; $\times 40$. 


\section{Decorin and B. Burgdorferi Colocalize in the Tunica Adventitia and Myocardial Connective Tissue during Chronic Infection}

The observed microenvironmental specificity of persistent spirochetes in response to B. burgdorferi-specific antibody suggested differences in the composition of the tunica adventitia and myocardial connective tissue compared to the tunica media. These microenvironments in which persistent

Table 1 Microenvironmental distribution of Borrelia burgdoferi in the heart after antibody-mediated disease resolution

\begin{tabular}{llc}
\hline $\begin{array}{l}\text { Inoculum/immune } \\
\text { serum }{ }^{\mathrm{a}}\end{array}$ & \multicolumn{1}{c}{ Microenvironment } & $\begin{array}{c}\text { Mean spirochete burden } \\
\text { score } \pm \text { s.e.m. }\end{array}$ \\
\hline B31/B31 & Tunica adventitia & $0.2 \pm 0.1^{\mathrm{b}}$ \\
& Tunica media & C $^{\mathrm{c}}$ \\
& Myocardial connective & $0.1 \pm 0.1^{\mathrm{d}}$ \\
& tissues & \\
& & \\
B31/NMS & Tunica adventitia & $1.4 \pm 0.3^{\mathrm{b}, \mathrm{c}, \mathrm{d}}$ \\
& Tunica media & $0.3 \pm 0.1$ \\
& Myocardial connective & $1.8 \pm 0.4^{\mathrm{b}, \mathrm{c}, \mathrm{d}}$ \\
& tissues &
\end{tabular}

Abbreviation: NMS, normal mouse serum.

Spirochete distribution and relative burden in the tunica adventitia, tunica media and myocardial connective tissue in $\mathrm{C} 3 \mathrm{H}$-scid mice at day 28 post inoculation.

${ }^{a}$ Mice were experimentally inoculated with B. burgdoferi strain B31 and passively immunized with either B31-specific immune serum or serum from uninfected naive mice.

b,c,d statistically significant differences (all $P$-values $\leq 0.05$ ) between respective B31 serum-immunized and NMS treatment groups in specific microenvironments are identified by matching alphabets.
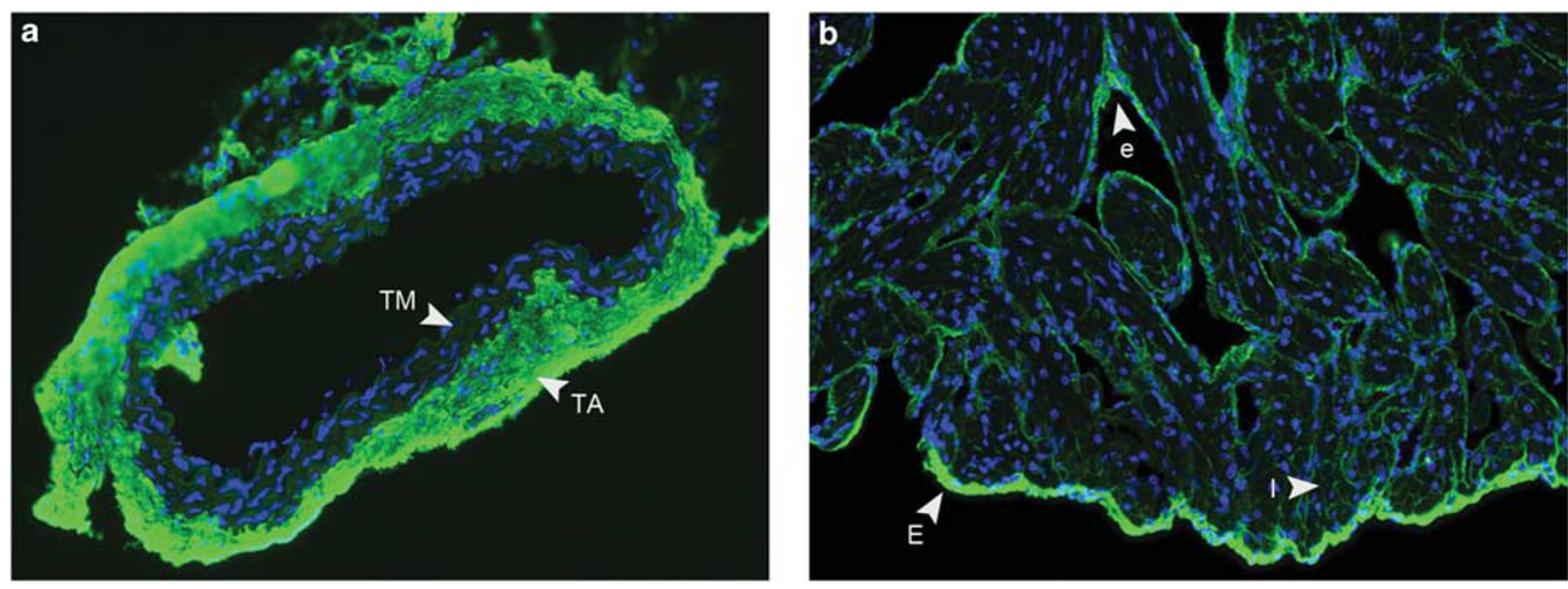

Figure 2 Immunolocalization of decorin within cardiovascular connective tissues. Decorin was localized to (a) the tunica adventitia (TA) of the heart base aorta, and (b) the epicardium (E), endocardium (e) and myocardial interstitium (I) of the heart. Complete absence of immunofluorescence (IF) for decorin in the tunica media (TM) of the heart base aorta highlighted the close apposition of these decorin-rich and decorin-poor microenvironments. IF with goat anti-decorin primary antibody and NL493 fluorochrome-labelled secondary donkey anti-goat antibody; DAPI counterstain; $\times 20$. 
connective tissue only) was unchanged between naive mice and chronically infected $\mathrm{C} 3 \mathrm{H}$ mice from the earlier study (Figure 3c). Microenvironmental colocalization of spirochetes and decorin (Figure 4) in the chronic stage of infection was demonstrated, further implicating decorin as a determining factor for defining permissive microenvironments. Based on these observations, B. burgdorferi preferentially localizes to connective tissue during early and chronic phases of infection, and decorin, not type I collagen, defines the connective-tissue microenvironment for persistence.

\section{Connective-Tissue Localization in Chronic Lyme Borreliosis is Dynamic}

Within a discourse on the pathogenesis of chronic bacterial infections, Godfrey ${ }^{45}$ proposed that localization of microorganisms is dynamic, with bouts of acute infection arising from persistent and protected reservoirs to repopulate previously cleared and less-protected microenvironments. Microenvironmental localization in decorin-rich connective tissues could be an end-stage event, driven by humoral immunity and maintained as a static process. Alternatively, microenvironmental localization could be a dynamic event, as proposed by Godfrey. ${ }^{45}$ To evaluate the dynamics of microenvironmental localization of $B$. burgdorferi in response to humoral immunity, the duration of infection in $\mathrm{C} 3 \mathrm{H}$-scid mice passively immunized with immune serum was extended to coincide with the time-dependent attrition of passively transferred immunoglobulins and thus, fading of humoral immunity. Thirty $\mathrm{C} 3 \mathrm{H}$-scid were inoculated with B. burgdorferi $\mathrm{cN} 40$ and 15 of these $30 \mathrm{C} 3 \mathrm{H}$-scid mice were
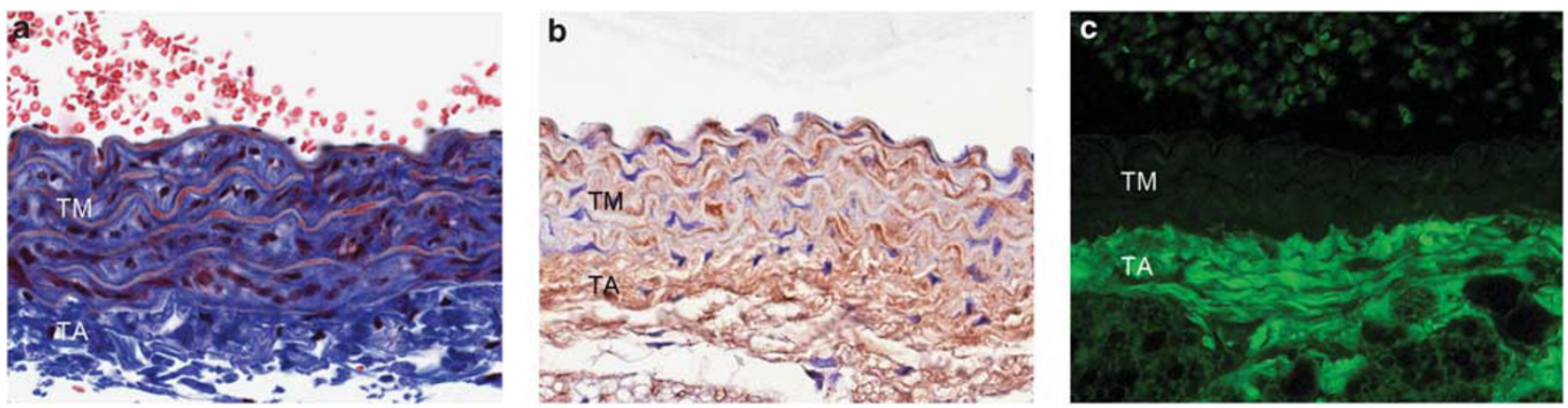

Figure 3 Distribution of connective tissue (a), type I collagen (b), and decorin (c) in the heart base aorta of a C3H mouse, chronically infected (60 days post inoculation) with $B$. burgdorferi. Collagen-rich connective tissues and type I collagen were broadly distributed throughout the tunica adventitia (TA) and tunica media (TM). Decorin was restricted to the TA. Masson's trichrome (a), indirect IHC with polyclonal rabbit anti-type I collagen antibody and immunoperoxidase reaction with 3, 3'-diaminiobenzidine (DAB) substrate (b), and IF with goat anti-decorin primary antibody and NL493 fluorochrome-labelled secondary donkey anti-goat antibody (c); $\times 20$.
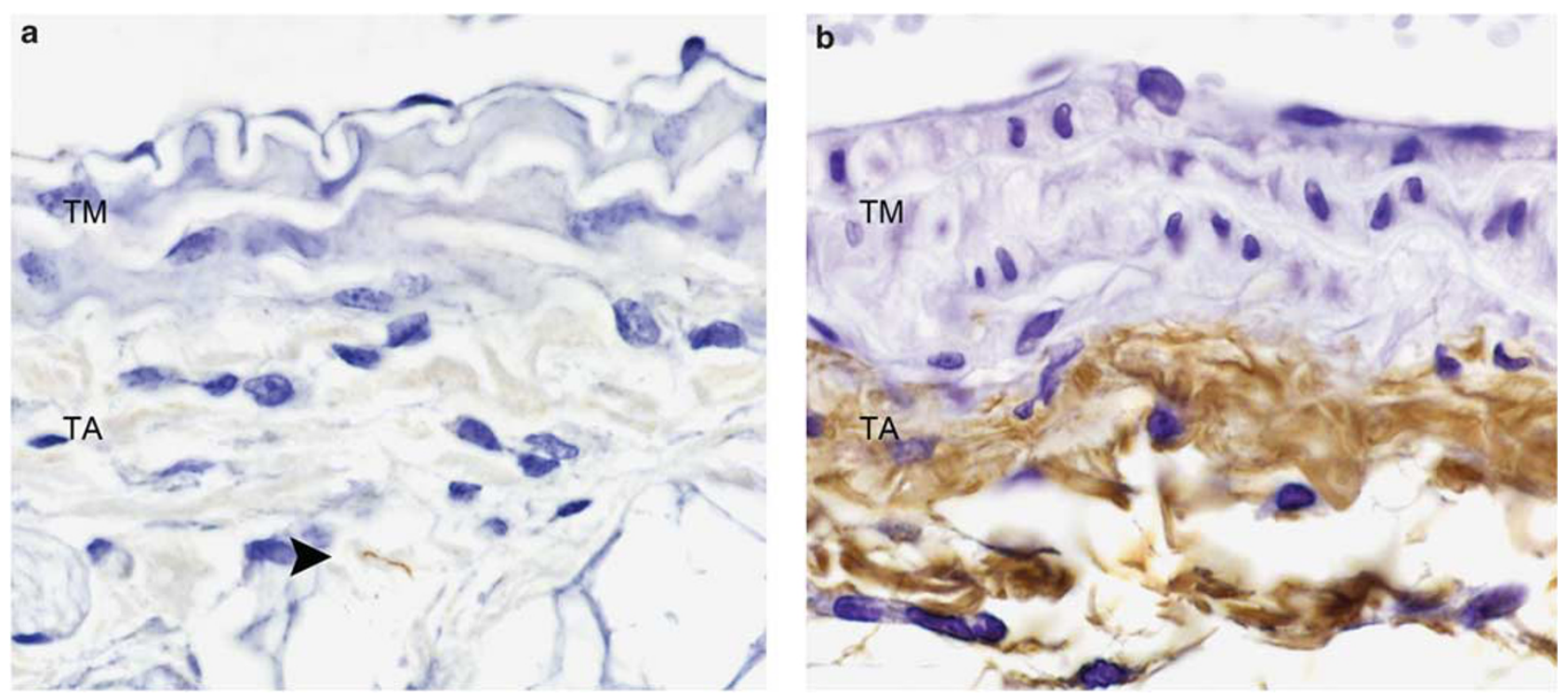

Figure 4 Colocalization of persistent B. burgdorferi and decorin during chronic infection of an immunocompetent $\mathrm{C} 3 \mathrm{H}$ mouse, at day 60 post inoculation. A single spirochete was identified in the tunica adventitia (a) of the heart base aorta (arrowhead). Restriction of decorin distribution to the tunica adventitia (b) remained constant over the duration of borrelial infection. Indirect IHC with rabbit anti-B. burgdorferi immune serum or goat anti-decorin antibody and immunoperoxidase reaction with 3, 3'-diaminiobenzidine (DAB) substrate; hematoxylin counterstain; $\times 100$. 
passively immunized with either cN40-specific immune serum or NMS from uninfected mice. Subsets of five mice from each group were necropsied on days 28,56 , and 72 post inoculation ( 4,32 , and 48 days post serum administration). Day 72 was arbitrarily identified as the maximum duration of the experiment, based on progressively severe arthritis in $\mathrm{C} 3 \mathrm{H}$-scid mice administered NMS.

Total serum IgG levels (Figure 5a) progressively declined over time in the mice administered NMS (negative control group) and the mice passively immunized with N40-specific immune serum. Total serum IgG levels were consistently lower to undetectable in the negative control group. B. burgdorferi N40-specific antibody levels (Figure $5 \mathrm{~b}$ ) at day 28 were statistically significantly greater $(P \leq 0.05)$ than at days 56 and 72 in passively immunized mice, confirming successful transfer of $B$. burgdorferispecific antibodies. At days 56 and 72, B. burgdorferi N40-specific antibody levels were near the minimum detection level and not significantly different from mice administered NMS. Therefore, the day 28 interval represented the established immune response and the day 56 and day 72 intervals represented decline of the B. burgdorferi-specific humoral immune response.

Passively transferred immune serum reduced, but did not clear, tissue spirochete burdens (Figures $6 \mathrm{a}-\mathrm{e}$ ) in the $\mathrm{C} 3 \mathrm{H}$ scid mice. At the initial time point, tissue burdens of spirochetes, based on copy numbers of flaB DNA per mg of tissue, in the passively immunized $\mathrm{C} 3 \mathrm{H}$-scid mice were lower than in NMS-treated C3H-scid mice. Over time, and with the waning of the antibody titer, tissue spirochete burdens proceeded to rise. The tissue spirochete burdens at the later time points, compared to the initial time point (day 56 and 72 versus day 28), were only significantly different in skin where tissue spirochete burdens were statistically significantly greater (Figure 6e). In NMS-treated $\mathrm{C} 3 \mathrm{H}$-scid mice, tissue burdens of spirochetes were initially higher, continued to progressively increase over time, and in many tissues, were significantly greater than in the passively immunized $\mathrm{C} 3 \mathrm{H}$-scid mice (Figures 6a, $\mathrm{b}$ and $\mathrm{d}$ ).

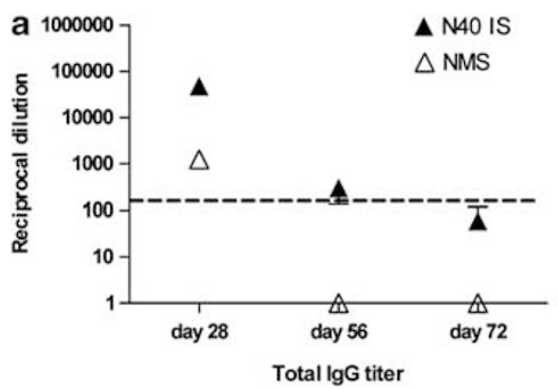

At day 28 in the $\mathrm{C} 3 \mathrm{H}$-scid mice passively immunized with immune serum, spirochetes were identified by IHC within the tunica adventitia only (Table 2). No spirochetes were identifiable within the tunica media or the myocardial connective tissues. At day 28, the mean spirochete burden scores in all microenvironments were statistically significantly lower (all $P$-values $\leq 0.05$ ) in mice treated with immune serum than in at least two of three microenvironments at all time points in the NMS-treated mice. In $\mathrm{C} 3 \mathrm{H}$-scid mice treated with immune serum, spirochetes were identified on days 56 and 72 within the previously cleared tunica media and the myocardial connective tissues. Identification of spirochetes in the tunica media on days 56 and 72 in $\mathrm{C} 3 \mathrm{H}$-scid mice treated with immune serum (Table 2) coincided with the decline of $B$. burgdorferi-specific antibody levels (Figure $5 b$ ). Though not significantly different from day 28 , identification of spirochetes in the tunica media at days 56 and 72 was interpreted as repopulation of a previously cleared microenvironment. All microenvironments, especially the tunica media (Figure 7), at all time points were heavily populated by spirochetes in mice administered NMS, suggesting that one permissive condition for tissue colonization is the absence of borrelia-specific antibody. Thus, the data (repopulation of previously cleared microenvironments, rising spirochete tissue burden by flaB DNA copy number, and coincident decline in borreliaspecific antibody titer) suggest that recrudescence and re-invasion of previously cleared microenvironments occurs with waning humoral immunity.

\section{DISCUSSION}

Using the heart as a tissue for investigation in the mouse model of chronic Lyme borreliosis, this study demonstrated a B. burgdorferi-specific antibody-driven change in microenvironmental localization of persisting spirochetes. Moreover, the antibody-driven change in borrelial microenvironmental sequestration was shown to be dynamic. With a decline of humoral immune pressure, recrudescence of persistent spirochetes resulted in the repopulation of

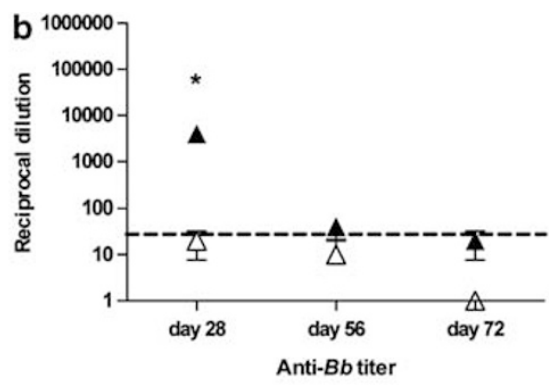

Figure 5 Total IgG (a) and B. burgdorferi-specific (b) antibody titers waned over time in C3H-scid mice passively immunized with B. burgdorferi strain CN40-specific immune serum (N40 IS). Mice were inoculated with $B$. burgdorferi strain $\mathrm{CN} 40$ and immune serum was last administered on day 24 post inoculation. Borrelia-specific serum titers were significantly greater $\left({ }^{*} P \leq 0.05\right)$ at day 28 but not significantly different from the negative control group (administered serum from naive uninfected mice, normal mouse serum (NMS)) at and after day 56 post inoculation. Each data point represents mean reciprocal dilution factor \pm s.e.m. from 4-5 mice. 

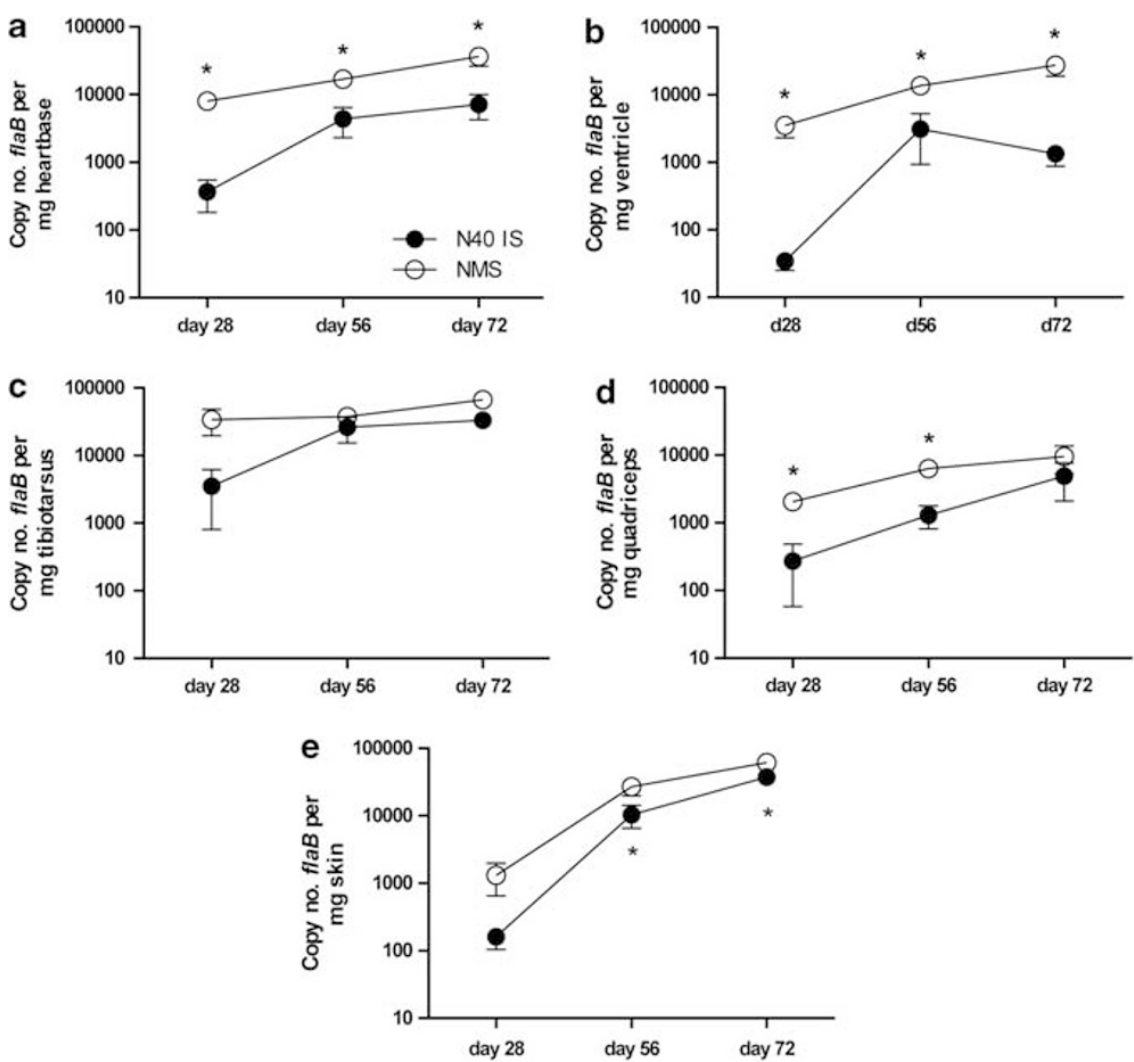

Figure 6 Recrudescence of $B$. burgdorferi as the B. burgdorferi-specific antibody titer waned at and after day 56 post inoculation in passively immunized $\mathrm{C} 3 \mathrm{H}$-scid mice. The $\mathrm{C} 3 \mathrm{H}$-scid mice were inoculated with $B$. burgdorferi strain $\mathrm{CN} 40$ (N40 IS) and were administered cN40-specific immune serum or serum from naive uninfected mice (NMS) in three doses, ending on day 24 post inoculation. Tissue spirochete burdens (as the mean copy number flaB DNA \pm s.e.m.) rose in the heart base (a), ventricle (b), tibiotarsus (c), quadriceps (d) and skin (e) as the antibody titer waned in the N40 IS-treated group, but only in skin (e) was the rise of spirochete tissue burdens statistically significant (*all $P$ values $\leq 0.05$ ). As expected, in NMS-treated mice (negative control group) tissue spirochete burdens were statistically significantly greater in the heart base (a) ( ${ }^{*}$ day $28, P<0.013$; day $56, P<0.004$; day 72 , $P<0.024)$, ventricle (b) (*day $28, P<0.04$; day 56, $P<0.007$; day $72, P<0.014$ ) and quadriceps (d) $(*$ day $28, P<0.004 ;$ day $56, P<0.002$ ).

antibody-cleared microenvironments. Finally, in this study, the recognized preferential connective-tissue localization of B. burgdorferi was confirmed and additional data were shown to support decorin as a significant determining factor of microenvironmental specificity.

The mechanism by which decorin defines protective microenvironments and prevents antibody clearance could be specific (due to antigen masking by binding to an immunogenic borrelial adhesin) or non-specific (by inhibiting antibody accessibility). Current evidence, including observations made in this study, supports the former possibility. A direct correlation between decorin expression in the homogenates of tissues and spirochete persistence has been observed in mice experimentally inoculated with B. burgdorferi, ${ }^{27}$ but that study did not localize the specificity of the microenvironment. Greater tissue decorin expression levels were reported in sites (joint and skin) that harbored greater spirochete burdens during chronic infection $(\sim 60$ days) or after passive immunization with $B$. burgdorferispecific immune serum. Decorin appeared to be the specific key component to these sites of sequestration, as these same sites harbored significantly fewer spirochetes (by copy numbers of flaB DNA) in chronically infected decorindeficient mice. Localization in these tissues could be driven by passive immunization of $\mathrm{C} 3 \mathrm{H}$-scid mice with polyclonal antibody to recombinant DbpA alone, ${ }^{6}$ and these sites failed to protect spirochetes that expressed non-decorin-binding immunogenic adhesins (OspA and $\mathrm{OspC}$ ), ${ }^{27,46}$ indicating that the protective capacity of these tissues was not due to non-specific impermeability to antibody but was specific for Dbp-expressing spirochetes. Our findings further prove the importance of the decorin-spirochete colocalization, and extend this observation, showing that intact spirochetes were present within decorin-rich microenvironments and there is tissue redistribution with fluctuations in humoral immune pressure.

The role of Dbps and their specific interaction with the ECM and decorin in the pathogenesis of Lyme disease has been extensively investigated. The Dbps include a pair of outer surface proteins, $\mathrm{DbpA}$ and $\mathrm{DbpB}$, that are constitutively expressed throughout all stages of infection, ${ }^{33,34,38,47}$ are surface-exposed, ${ }^{19,38}$ and are highly immunogenic..$^{38,47,48}$ 
Table 2 Loss of specific decorin-dependent connective-tissue localization during recrudescence of Borrelia burgdorferi

Mean spirochete burden

score \pm s.e.m

\begin{tabular}{llccc} 
Inoculum/immune serum $^{\mathrm{a}}$ & Microenvironment & Day 28 & Day 56 & Day 72 \\
\hline N40/N40 & Tunica adventitia & $0.5 \pm 0.3$ & 1.0 & 1.0 \\
& Tunica media & $-{ }^{\mathrm{b}}$ & $1.2 \pm 0.5$ & $0.8 \pm 0.4$ \\
& Myocardial connective & - & 1.0 & $0.9 \pm 0.2$ \\
& tissues & & & \\
& & & & \\
N40/NMS & Tunica adventitia & $1.8 \pm 0.2$ & 1.0 & $1.6 \pm 0.3$ \\
& Tunica media & $1.0 \pm 0.3$ & $2.8 \pm 0.2^{\mathrm{b}}$ & $3^{\mathrm{b}}$ \\
& Myocardial connective & $1.4 \pm 0.4$ & $1.6 \pm 0.3$ & $1.2 \pm 0.2$ \\
& tissues & & &
\end{tabular}

Abbreviation: NMS, normal mouse serum.

As humoral immunity wanes, the previously cleared decorin-poor tunica media is repopulated.

${ }^{\mathrm{a}}$ Spirochete distribution and relative burden in $\mathrm{C} 3 \mathrm{H}$-scid mice at days 28,56 and 72 post inoculation, experimentally inoculated with Borrelia burgdoferi strain cN40 and passively immunized with cN40-specific immune serum or serum from uninfected naive mice.

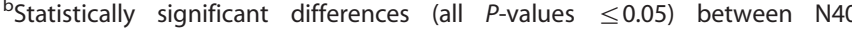
serum-immunized and NMS treatment groups within the respective microenvironments and durations of infection identified by matching alphabet.

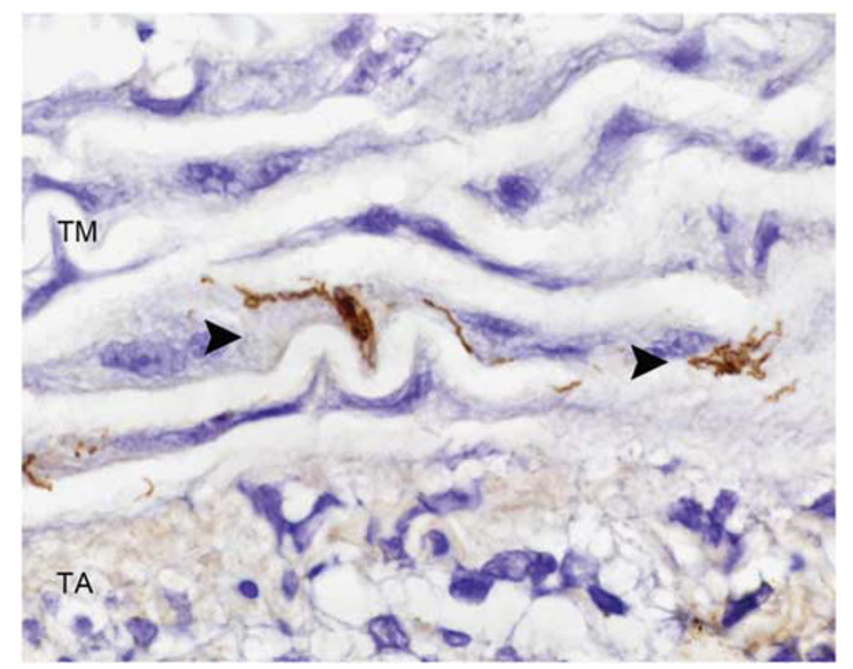

Figure 7 Colonization of the decorin-poor tunica media (TM) by B. burgdorferi in a $\mathrm{C} 3 \mathrm{H}$-scid mouse administered with normal mouse serum (NMS), at day 72 post inoculation. Both single spirochetes and microcolonies (arrowheads) were present. Spirochetes also populated the tunica adventitia (TA). Indirect IHC with rabbit anti-B. burgdorferi immune serum and immunoperoxidase reaction with $3,3^{\prime}$-diaminiobenzidine (DAB) substrate; hematoxylin counterstain; $\times 40$.

DbpA is considered to be the more biologically relevant adhesin, as it has a stronger specificity for the host ligand decorin, ${ }^{19}$ induces stronger protective immunity, ${ }^{34,38}$ can solely induce a disease-resolving immune response, ${ }^{6}$ and can restore a wild-type phenotype to Dbp-deficient mutant Borrelia. ${ }^{49-51}$ Deficiency in Dbps results in a phenotype of delayed dissemination with an overall decrease in Dbp-deficient spirochete tissue burdens ${ }^{51,52}$ and decreased retrieval of Dbp-deficient spirochetes from tissues distant to the inoculation site ${ }^{18,19,25}$ at early time points ( $<30$ days). However, in the later stages of infection ( $>42$ days), tissue spirochete levels equivalent to wild-type infection are eventually reached and persistence is achieved by the Dbp-deficient spirochetes. ${ }^{51}$ Thus, Dbps are involved in dissemination, are not absolutely required for persistence, and the phenotype of Dbp-deficient mutant Borrelia suggests the existence of functionally redundant adhesins that are involved in but less efficient at dissemination.

The lack of an absolute Dbp requirement for persistence does not negate the potential importance of the Dbp-decorin interaction in immune evasion. Rather, genetically manipulated spirochetes that lack a highly immunogenic, continuously expressed outer surface protein would be expected to evade immune clearance and persist. This scenario would be more likely to occur if there existed functionally redundant adhesins that may be less effective, but are not as immunogenic. Overproduction of either DbpA or DbpB has shown the opposite immunogenic pattern, with generation of a faster and stronger specific humoral immune response $e^{50,53}$ and increased binding of specific antibodies to spirochetes. ${ }^{50}$ Despite this, DbpA- and DbpB-overexpressing spirochetes are still not cleared and persist in a manner equivalent to the rescued wild-type phenotype of DbpA/B-complemented spirochetes. ${ }^{50}$ In a separate study, spirochetes were shown to persist in lesser numbers and, predominantly, in the skin. ${ }^{53}$ The DbpA-overexpressing mutant spirochetes share a phenotype of delayed dissemination with Dbp-deficient mutant spirochetes. ${ }^{50,53}$ As one could postulate that the Dbp-deficient spirochetes are disseminating with the use of less-efficient functionally redundant adhesins, so, one could posit that the Dbp-overexpressing Borrelia may be slowed by increased interaction with the ECM. Increased interaction with the ECM (and decorin, specifically) may also explain why these extremely immunogenic, adhesin-overexpressing Borrelia may be able to avoid antibody-mediated clearance.

This study highlights the importance of the antigenspecific antibody response in Lyme borreliosis (reviewed by La Rocca $e a l^{54}$ ). The biphasic patterns of sequestration and subsequent recrudescence simultaneously occurring with the rise and fall of the B. burgdorferi-specific antibody titer suggest that the dynamics of persistence are at least partially dependent on humoral immune pressure. A similar phenomenon of recrudescence has been reported following passive transfer of neutralizing $B$. burgdorferi antigen-specific antibodies in immunodeficient mice. ${ }^{28}$ The aforementioned study also demonstrated antibody-driven, antigen-dependent (OspC) positive selection of non-antigen expressing spirochetes with reversion to antigen expression after 
antibody neutralization. ${ }^{28}$ The antibody-driven immune pressure either induced antigen downregulation or selected for a specific sub-population of B. burgdorferi that was then able to persist. A very similar phenomenon occurs in other borrelial species, such as the relapsing fever Borrelia spp., with cyclical proliferation and antibody clearance of one dominant serotype, only to allow another serotype to then proliferate. ${ }^{54}$

In this study, the phenomenon of recrudescence presented in the passively immunized $\mathrm{C} 3 \mathrm{H}$-scid mouse model is likely to be an exaggeration of what may occur in spontaneous infections. The biological validity of local recrudescence is supported by both clinical and experimental evidence of a cyclical disease pattern and a hyperimmunization antibody profile (suggestive of repetitive exposure) to chronic human and murine Lyme borreliosis., ${ }^{1,7}$ The natural antibody profile reveals the caveats to local recrudescence in spontaneous Lyme disease. Bouts of untreated human Lyme arthritis typically occur in the face of a high antibody $\operatorname{titer}^{7,8}$ suggesting that fluctuation in the quality of the antibody response, rather than quantity, is the determining factor for spirochetal repopulation of previously cleared microenvironments. Other cofactors, such as micro-trauma, within chronically infected joints may influence the episodic nature of clinical disease. Thus, we simply conclude that fluctuations in antibody-mediated immune pressure and recurrent bouts of local recrudescence arising from an internal reservoir of persistent bacteria may be one necessary factor in the maintenance of chronic borreliosis. ${ }^{45,55}$

\section{ACKNOWLEDGEMENTS}

We thank Kimberley Olsen, Edlin Escobar and Diane Naydan for their technical expertise and advice. This study was supported in part by $\mathrm{NIH}$ grants T32 Al 06055 (DI), T32 OD 011147 (DI), and R01 Al 26815 (SWB, SF, $\mathrm{EH}, \mathrm{KO})$.

\section{DISCLOSURE/CONFLICT OF INTEREST}

The authors declare no conflict of interest.

1. Steere AC, Coburn J, Glickstein L. The emergence of Lyme disease. J Clin Invest 2004;113:1093-1101.

2. Cabello FC, Godfrey HP, Newman SA. Hidden in plain sight: Borrelia burgdorferi and the extracellular matrix. Trends Microbiol 2007;15: 350-354.

3. Duray PH, Steere AC. Clinical pathologic correlations of Lyme disease by stage. Ann NY Acad Sci 1988;539:65-79.

4. Barthold SW, Persing $\mathrm{DH}$, Armstrong $\mathrm{AL}$, et al. Kinetics of Borrelia burgdorferi dissemination and evolution of disease after intradermal inoculation of mice. Am J Pathol 1991;139:263-273.

5. Shih CM, Pollack RJ, Telford SR, et al. Delayed dissemination of Lyme disease spirochetes from the site of deposition in the skin of mice. J Infect Dis 1992;166:827-831.

6. Barthold SW, Hodzic E, Tunev S, et al. Antibody-mediated disease remission in the mouse model of Lyme borreliosis. Infect Immun 2006;74:4817-4825.

7. Barthold SW, de Souza MS, Janotka JL, et al. Chronic Lyme borreliosis in the laboratory mouse. Am J Pathol 1993;143:959-971.

8. Schoen RT. A case revealing the natural history of untreated Lyme disease. Nat Rev Rheumatol 2011;7:179-184.
9. Nocton JJ, Dressler F, Rutledge BJ, et al. Detection of Borrelia burgdorferi DNA by polymerase chain reaction in synovial fluid from patients with Lyme arthritis. N Engl J Med 1994;330:229-234.

10. Li X, McHugh GA, Damle N, et al. Burden and viability of Borrelia burgdorferi in skin and joints of patients with erythema migrans or Lyme arthritis. Arthritis Rheum 2011;63:2238-2247.

11. Armstrong AL, Barthold SW, Persing DH, et al. Carditis in Lyme disease susceptible and resistant strains of laboratory mice infected with Borrelia burgdorferi. Am J Trop Med Hyg 1992;47:249-258.

12. Cadavid D, O'Neill $\mathrm{T}$, Schaefer $\mathrm{H}$, et al. Localization of Borrelia burgdorferi in the nervous system and other organs in the nonhuman primate model of Lyme disease. Lab Invest 2000;80:1043-1054.

13. Cadavid $D$, Bai $Y$, Hodzic $E$, et al. Cardiac involvement in non-human primates infected with the Lyme disease spirochete Borrelia burgdorferi. Lab Invest 2004;84:1439-1450.

14. Imai DM, Barr BC, Daft B, et al. Lyme neuroborreliosis in 2 horses. Vet Pathol 2011;48:1151-1157.

15. Alberts B, Johnson A, Lewis J, et al. Molecular Biology of the Cell. 4th edn, New York, NY, USA, 2002.

16. Finlay BB, Falkow S. Common themes in microbial pathogenicity revisited. Microbiol Mol Biol Rev 1997;61:136-169.

17. Antonara S, Ristow L, Coburn J. Adhesion mechanisms of Borrelia burgdorferi. Adv Exp Med Biol 2011;715:35-49.

18. Guo BP, Norris SJ, Rosenberg LC, et al. Adherence of Borrelia burgdorferi to the proteoglycan decorin. Infect Immun 1995;63: 3467-3472, 33.

19. Guo BP, Brown EL, Dorward DW, et al. Decorin-binding adhesins from Borrelia burgdorferi. Mol Microbiol 1998;30:711-723, 38.

20. Parveen $\mathrm{N}$, Leong JM. Identification of a candidate glycosaminoglycan-binding adhesin of the Lyme disease spirochete Borrelia burgdorferi. Mol Microbiol 2000;35:1220-1234.

21. Brissette CA, Bykowski T, Cooley AE, et al. Borrelia burgdorferi RevA antigen binds host fibronectin. Infect Immun 2009a;77:2802-2812.

22. Verma A, Brissette CA, Bowman A, et al. Borrelia burgdorferi BmpA is a laminin-binding protein. Infect Immun 2009;77:4940-4946.

23. Brissette CA, Verma A, Bowman $A$, et al. The Borrelia burgdorferi outer-surface protein ErpX binds mammalian laminin. Microbiology 2009b;155:863-872.

24. Coburn J, Chege W, Magoun L, et al. Characterization of a candidate Borrelia burgdorferi b3-chain integrin ligand identified using phage display library. Mol Microbiol 1999;34:9266-9940.

25. Zambrano MC, Beklemisheva AA, Bryksin AV, et al. Borrelia burgdorferi binds to, invades, and colonizes native type I collagen lattices. Infect Immun 2004;72:3138-3146, 34.

26. Antonara S, Chafel RM, LaFrance $\mathrm{M}$, et al. Borrelia burgdorferi adhesins identified using in vivo phage display. Mol Microbiol 2007;66: 262-276.

27. Liang FT, Brown EL, Wang $\mathrm{T}$, et al. Protective niche for Borrelia burgdorferi to evade humoral immunity. Am J Pathol 2004;165: 977-985.

28. Liang FT, Jacobs MB, Bowers LC, et al. An immune evasion mechanism for spirochetal persistence in Lyme borreliosis. J Exp Med 2002;195:415-422.

29. Barthold SW, Feng S, Bockenstedt LK, et al. Protective and arthritisresolving activity in sera of mice infected with Borrelia burgdorferi. Clin Infect Dis 1997;25:S9-S17.

30. Casjens S, Palmer N, van Vugt $\mathrm{R}$, et al. A bacterial genome in flux: the twelve linear and nine circular extrachromosomal DNAs in an infectious isolate of the Lyme disease spirochete Borrelia burgdorferi. Mol Microbiol 2000;35:490-516.

31. Fraser CM, Casjens S, Huang WM, et al. Genomic sequence of a Lyme disease spirochaete, Borrelia burgdorferi. Nature 1997;390:580-586.

32. Barbour AG. Isolation and cultivation of Lyme disease spirochetes. Yale J Biol Med 1984;57:521-525.

33. Hodzic E, Feng S, Freet KJ, et al. Borrelia burgdorferi population dynamics and prototype gene expression during infection of immunocompetent and immunodeficient mice. Infect Immun 2003;71:5042-5055.

34. Feng $\mathrm{S}$, Hodzic E, Stevenson B, et al. Humoral immunity to Borrelia burgdorferi N40 decorin binding proteins during infection in laboratory mice. Infect Immun 1998;66:2827-2835.

35. Barthold SW, deSouza M, Feng S. Serum-mediated resolution of Lyme arthritis in mice. Lab Invest 1996;74:57-67. 
36. Roberts WC, Mullikin BA, Lathigra $\mathrm{R}$, et al. Molecular analysis of sequence heterogeneity among genes encoding decorin binding proteins A and B of Borrelia burgdorferi sensu lato. Infect Immun 1998;66:5275-5285

37. Benoit VM, Fischer JR, Lin YP, et al. Allelic variation of the Lyme disease spirochete adhesin DbpA influences spirochetal binding to decorin, dermatan sulfate, and mammalian cells. Infect Immun 2011;79:3501-3509.

38. Hanson MS, Cassatt DR, Guo BP, et al. Active and passive immunity against Borrelia burgdorferi decorin binding protein $\mathrm{A}(\mathrm{DbpA})$ protects against infection. Infect Immun 1998;66:2143-2153.

39. Danielson KG, Baribault H, Holmes DF, et al. Targeted disruption of decorin leads to abnormal collagen fibril morphology and skin fragility. J Cell Biol 1997;136:729-743.

40. Reissen $R$, Isner JM, Blessing $E$, et al. Regional differences in the distribution of the proteoglycans biglycan and decorin in the extracellular matrix of atherosclerotic and restenotic human coronary arteries. Am J Pathol 1994;144:962-974.

41. Farquharson C, Robins SC. Immunolocalization of collagen types I and III in the arterial wall of the heart. Histochem J 1989;21:172-178.

42. Adhikari N, Carlson M, Lerman B, et al. Changes in expression of proteoglycan core proteins and heparin sulfate enzymes in the developing and adult murine aorta. J Cardiovasc Trans Res 2011;4:313-320.

43. Seidler DG, Dreier R. Decorin and its galactosaminoglycan chain: Extracellular regulator of cellular function? IUBMB Life 2008;60: 729-733.

44. Bianco P, Fisher LW, Young MF, et al. Expression and localization of the two small proteoglycans biglycan and decorin in developing human skeletal and non-skeletal tissues. J Histochem Cytochem 1990;38: 1549-1563.

45. Godfrey HP. Pathogenesis of chronic bacterial infections. Trends Microbiol 1998;6:303.
46. Strother KO, Hodzic E, Barthold SW, et al. Infection of mice with Lyme disease spirochetes constituitively produducing outer surface proteins A and B. Infect Immun 2007;75:2786-2794.

47. Cassatt DR, Patel NK, Ulbrandt ND, et al. DbpA, but not OspA, is expressed by Borrelia burgdorferi during spirochetemia and is a target for protective antibodies. Infect Immun 1998;66: 5379-5387.

48. Tunev SS, Hastey CJ, Hodzic E, et al. Lymphadenopathy during Lyme borreliosis is caused by spirochete migration-induced specific B cell activation. PLoS Pathog 2011;7:e1002066.

49. Shi $Y, X u Q, M c S h a n ~ K$, et al. Both decorin-binding proteins $A$ and $B$ are critical for the overall virulence of Borrelia burgdorferi. Infect Immun 2008a;76:1239-1246.

50. Shi Y, Xu Q, Seemanaplli SV, et al. Common and unique contributions of decorin-binding proteins $A$ and $B$ to the overall virulence of Borrelia burgdorferi. Plos One 2008b;3:e3340.

51. Imai DM, Samuels DS, Feng $S$, et al. The early dissemination defect attributed to disruption of decorin-binding proteins is abolished in chronic murine Lyme borreliosis. Infect Immun 2013;81: 1663-1673.

52. Weening EH, Parveen N, Trzeciakowski JP, et al. Borrelia burgdorferi lacking DbpBA exhibits an early survival defect during experimental infection. Infect Immun 2008;76:5694-5705.

53. Xu Q, Seemanaplli SV, McShan K, et al. Increasing the interaction of Borrelia burgdorferi with decorin significantly reduces the 50 percent infectious dose and severely impairs dissemination. Infect Immun 2007;75:4272-4281.

54. LaRocca TJ, Benach JL. The important and diverse roles of antibodies in the host response to Borrelia infections. Curr Top Microbiol Immunol 2008;319:63-103.

55. Young D, Hussell T, Dougan G. Chronic bacterial infections: living with unwanted guests. Nat Immunol 2002;3:1026-1032. 